\title{
Chronic renal failure and tuberous sclerosis: a rare association
}

\author{
A.B. Nimr, ${ }^{1}$ P.J. Patel, ${ }^{2}$ R.K. Tongia ${ }^{1}$ and F. Tamizuddin ${ }^{1}$ \\ Departments of 'Internal Medicine and ${ }^{2}$ Radiology, King Abdul Aziz University Hospital, King Saud University, \\ Saudi Arabia.
}

\begin{abstract}
Summary: A 38 year old Saudi patient with tuberous sclerosis and chronic renal failure is described. The association of these two conditions has rarely been described. This case supports the observation that when chronic renal failure occurs in patients with tuberous sclerosis, cystic renal disease (with or without hamartomas) is more likely to be the underlying cause, rather than hamartomas alone.
\end{abstract}

\section{Introduction}

There is a well established association between tuberous sclerosis and renal hamartomas ${ }^{1}$ but the incidence of chronic renal failure is rare. ${ }^{2}$ We would like to report a case of chronic renal failure due to tuberous sclerosis.

\section{Case report}

A 38 year old Saudi male, who was known to have tuberous sclerosis was admitted for the investigation of anaemia. His past medical history was unremarkable apart from seizures since childhood, the last of which was 6 years before admission.

On physical examination, he was edentulous with extensive small fibromata involving both the upper and lower ginginal margins. He demonstrated extensive typical cutaneous manifestations of tuberous sclerosis. Intellectual function was normal and he was normotensive.

Initial investigations showed hypochromic microcytic anaemia (haemoglobin $8.6 \mathrm{~g} / \mathrm{dl}$ ); subsequent investigations revealed no obvious cause for his anaemia apart from chronic renal failure (blood urea $9.9 \mathrm{mmol} / 1$, serum creatinine of $270 \mathrm{mmol} / 1$, creatinine clearance was $20 \mathrm{ml} / \mathrm{min}$ ). Urine examination showed proteinuria of $1 \mathrm{~g} / 24 \mathrm{~h}$.

An intravenous urogram showed bilaterally enlarged kidneys with poor visualization, while ultrasound of kidneys confirmed their enlargement with bilateral multiple cysts of variable sizes. The liver and spleen were normal. The patient discharged himself against medical advice. The final diagnosis was renal failure

Correspondence: P.J. Patel, M.D., D.M.R.D., F.R.C.R. (U.K.), Radiology Department (40), King Khalid University Hospital, P.O. Box 2925, Riyadh, 11461, Saudi Arabia.

Accepted: 3 March 1987 due to cystic kidney disease of tuberous sclerosis and iron deficiency anaemia.

Four months later, he was readmitted for reassessment, when his kidneys had become palpable. His haemoglobin had dropped to $6.6 \mathrm{~g} / \mathrm{dl}$ and his renal function deteriorated with a blood urea of 26.8 $\mathrm{mmol} / \mathrm{l}$, a serum creatinine of $451 \mathrm{mmol} / 1$ and a creatinine clearance of $14 \mathrm{ml} / \mathrm{min}$. Computed tomographic (CT) scan of the brain (Figure 1) demonstrated the characteristic periventricular calcification, while CT scan of the abdomen (Figure 2), with and without contrast, showed multiple cysts of variable sizes in both kidneys without evidence of hamartomas.

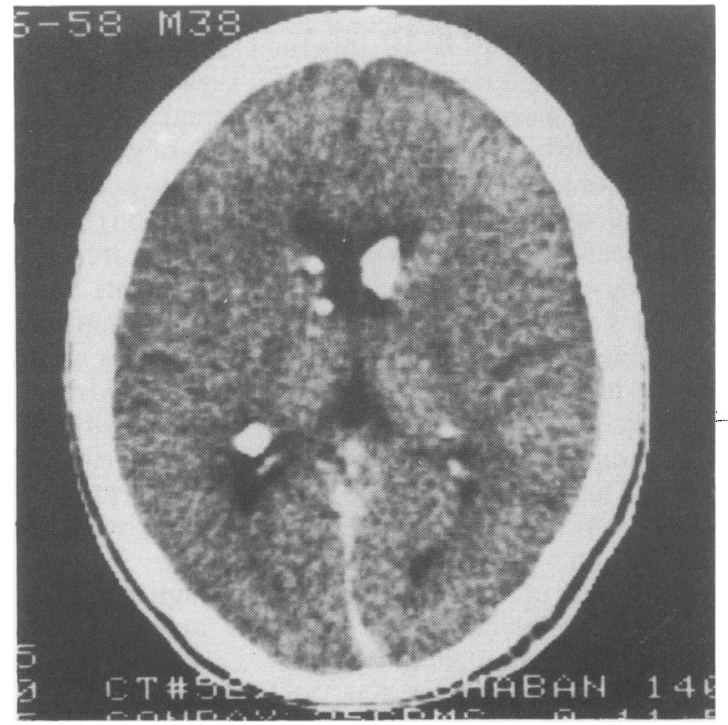

Figure 1 CT scan of brain showed bilateral periventricular calcification suggesting tuberous sclerosis.

(C) The Fellowship of Postgraduate Medicine, 1987 


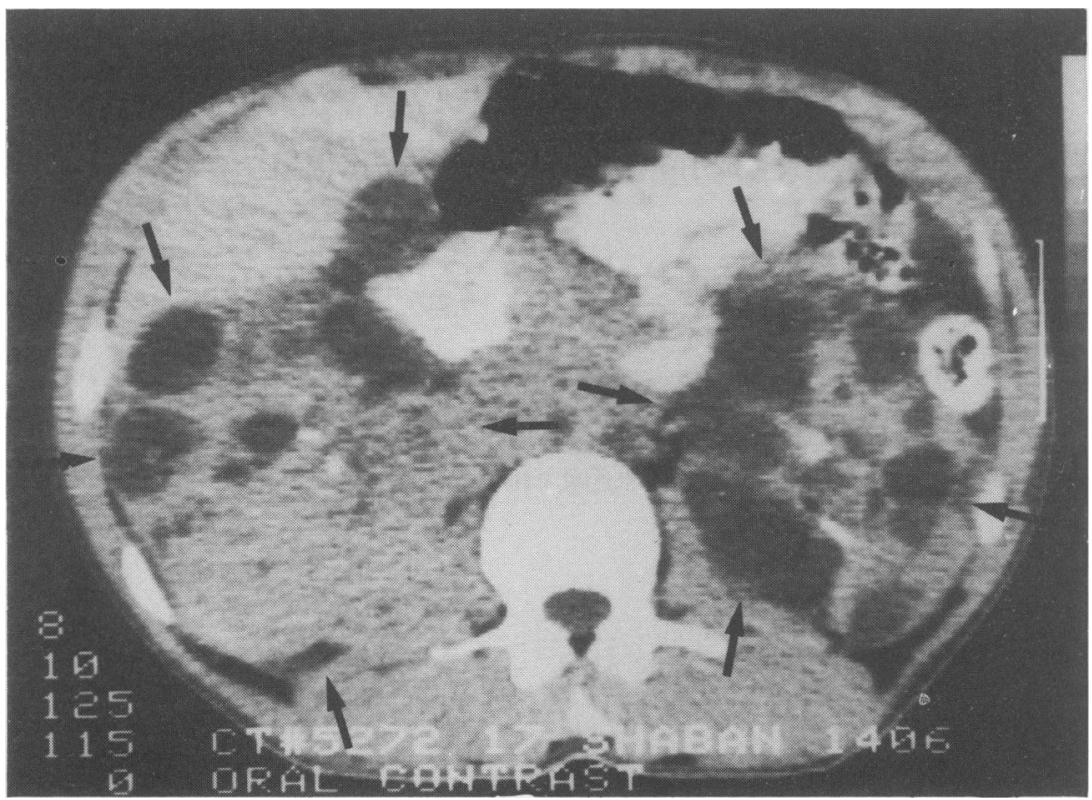

Figure 2 CT scan of abdomen showed enlargement of both kidneys with multiple cysts (arrows). There was no evidence of hamartomas.

The patient is being managed conservatively pending renal transplantation.

\section{Discussion}

Tuberous sclerosis is an inherited neurocutaneous disorder characterized by seizures, a variety of cutaneous lesions associated with visceral hamartomas and mental retardation, which occurs in $60 \%$ of patients. ${ }^{1}$ Renal hamartomas or angiomyolipomata occur in $50-80 \%$ of patients, but cystic renal lesions have rarely been described with tuberous sclerosis; rarer still is the development of renal failure in patients with tuberous sclerosis and isolated angiomyolipomata of the kidneys. ${ }^{3}$ It has been suggested that when renal failure develops in a patient with tuberous sclerosis, it is most likely that renal cysts (with or without hamartomas) are present, rather than hamartomas alone. ${ }^{2}$ Our case, whose renal lesion was confirmed by non-invasive radiological means only, supports this observation.

This rarity of renal failure despite the high incidence of renal involvement in tuberous sclerosis has previously been noted: in two large series, 29 patients reported by Crotchley $\& \mathrm{Earl}^{4}$ and 38 patients reported by Golji ${ }^{5}$ not a single case of chronic renal failure was observed. ${ }^{2}$ Indeed, only seven cases of chronic renal failure and tuberous sclerosis have so far been reported in the literature. ${ }^{2}$ This low incidence of chronic renal failure could be explained by the observation that $75 \%$ of patients with tuberous sclerosis die before the age of 20 years, because of seizures or neoplasia. ${ }^{1,2}$ Therefore as more attention is directed to the management of the neurological complications of this disorder, it is expected that more patients with renal impairment will be reported. The rapid deterioration of this patient is most probably due to rapid expansion of the cysts, with the kidneys becoming palpable within 4 months and with consequential parenchymal compression as explained by Okada et al. ${ }^{2}$

It was previously difficult to differentiate cystic lesions of tuberous sclerosis from those of polycystic kidney disease. ${ }^{1}$ However, with the help of ultrasound and high resolution CT scan these can now be distinguished. ${ }^{6}$ Moreover, renal biopsy offers a definitive diagnosis with the presence of eosinophilic hyperplastic changes in the walls of the cysts of tuberous sclerosis, and its absence in those of other polycystic kidney disease.

The presence of mild proteinuria and the absence of hypertension in our patient are noteworthy, and are consistent with previous observations. ${ }^{1}$

The practical implication of the occurrence of renal failure in patients with tuberous sclerosis is the unsuitability of some patients for chronic haemodialysis, since $60 \%$ of patients with tuberous sclerosis may have 
mental subnormality. ${ }^{1}$

Finally, in patients suspected of tuberous sclerosis, when history - which can be lacking in $50 \%$ of

\section{References}

1. Anderson, D. \& Tannen, R.L. Tuberous sclerosis and chronic renal failure. Am J Med 1969, 47: 163-168.

2. Okada, R.D., Platt, M.A. \& Fleishman, J. Chronic renal failure in patients with tuberous sclerosis: association with renal cysts. Nephron 1982, 30: 85-88.

3. Stapleton, F.B., Johnson, D., Kaplan, G. \& Griswold, W. The cystic renal lesion in tuberous sclerosis. $J$ Pediatr 1980, 97: 574-579. patients ${ }^{3}$ - and the clinical picture are not conclusive, CT scan of the kidneys and brain could be of great value in confirming the diagnosis.

4. Critchley, M. \& Earl, C.J.C. Tuberous sclerosis and allied conditions. Brain 1952, 55: $311-346$.

5. Golji, H. Tuberous sclerosis and renal neoplasms. J Urol 1961, 85: 919-923.

6. Yu, D.T. \& Sheth, J.K. Cystic renal involvement in tuberous sclerosis. Clin Pediatr 1985, 24: 36-38. 